\title{
Suplementación de clorhidrato de zilpaterol en corderos finalizados con dieta sin fibra de forraje
}

\author{
Ricardo Vicente-Pérez ${ }^{\text {a }}$ \\ Ulises Macías-Cruz ${ }^{\mathrm{b} *}$ \\ Ramón Andrade Mancilla ${ }^{\text {a }}$ \\ Rogelio Vicente ${ }^{a}$ \\ Enrique O. García ${ }^{\text {a }}$ \\ Ricardo Martínez ${ }^{\text {a }}$ \\ Leonel Avendaño-Reyes ${ }^{b}$ \\ Oziel D. Montañez ${ }^{c}$
}

${ }^{a}$ Universidad de Guadalajara, Departamento de Producción Agrícola-CUCSUR, Autlán de Navarro. Jalisco, México.

b Universidad Autónoma de Baja California, Instituto de Ciencias Agrícolas, Valle de Mexicali, Baja California, México.

c Universidad de Guadalajara, Departamento de Ciencias de la Naturaleza-CUSUR, Ciudad Guzmán. Jalisco, México.

* Autor de correspondencia: ulisesmacias1988@ hotmail.com

\section{Resumen:}

Un total de 24 corderos de pelo Kathadin se distribuyeron bajo un diseño de bloques completamente al azar en dos tratamientos para evaluar el efecto de suplementar clorhidrato de zilpaterol genérico (CZ; 0 vs $10 \mathrm{mg} / \mathrm{d} / \mathrm{animal}$ ) en una dieta de finalización sin fibra de forraje, sobre comportamiento productivo, características de la canal, rendimiento de cortes 
primarios y pesos de despojos expresados como un porcentaje del peso al sacrificio. Se realizó una prueba de comportamiento en corral de 30 días y después se sacrificó la mitad de los animales de cada tratamiento $(n=6)$. La suplementación del CZ no afectó la ganancia de peso, pero sí mejoró $(P \leq 0.02)$ la eficiencia alimenticia, el peso y rendimiento de canal, y el área del músculo Longissimus dorsi, así como el rendimiento de pierna y lomo completo. Tanto grasa KPH como mesentérica se redujeron $(P \leq 0.05)$ por efecto de $\mathrm{CZ}$. El resto de los porcentajes de despojos de la canal no fueron afectados por CZ. Se concluye que la suplementación dietaría de CZ genérico mejoró la deposición de masa muscular por reducir la deposición de grasa interna, favoreciendo la eficiencia alimenticia de cordero engordados con dieta sin fibra de forraje.

Palabras clave: Ovinos de pelo, Engorda de borregos, Agonista adrenérgico, Grofactor®.

Recibido: 14/11/2018

Aceptado: 1/07/2019

\section{Introducción}

El inventario ovino mexicano tuvo un incremento del $20 \%$ en la última década, lo que generó para el 2018 una producción de carne de 62,939 t y una disminución considerable en las importaciones de la misma ${ }^{(1)}$. La producción de carne de ovino muestra desde hace algunas décadas ser un nicho de oportunidad en el mercado mexicano y, en general, a través del mundo, ya que la demanda es insatisfecha y el kilogramo de carne tiene un precio competitivo $^{(2)}$. En este sentido, la búsqueda de estrategias que permitan mejorar la producción de carne ovina es una necesidad inminente en la actualidad.

El agonista adrenérgico $\beta 2$ (AA $\beta 2$ ) clorhidrato de zilpaterol (CZ) es un promotor de crecimiento que resulta efectivo para mejorar el comportamiento productivo y las características de la canal de importancia económica cuando se ofrece entre 4 y 5 semanas antes del sacrificio a corderos finalizados en corral ${ }^{(3-6)}$, no así en pastoreo ${ }^{(7)}$. Este AA $\beta 2$ funciona como promotor de crecimiento porque redistribuye sustrato energético del tejido graso y algunos órganos hacia la formación de masa muscular (hipertrofia) ${ }^{(4,8,9)}$. Cabe mencionar que la efectividad del CZ como promotor de crecimiento en corderos de engorda se ha demostrado ofreciendo dietas formuladas tanto con grano y forraje para favorecer el correcto funcionamiento ruminal. Sin embargo, en la actualidad se están formulando dietas 
para engorda de ovinos usando fuentes de fibras alternas al forraje, ya que es escaso en algunas regiones y épocas del año, así como en ocasiones tiene un costo elevado ${ }^{(10)}$.

El aserrín y los desechos de agroindustria son dos fuentes de fibra alterna que no provocan problemas digestivos en corderos al sustituir el $100 \%$ de la fracción de forraje en la dieta de engorda ${ }^{(11,12)}$. No obstante, no se ha determinado la efectividad del $\mathrm{CZ}$ como promotor de crecimiento en corderos alimentados sin fibra de forraje. Por lo tanto, el objetivo fue evaluar el efecto del $\mathrm{CZ}$ sobre comportamiento productivo, características de la canal y rendimiento de cortes primarios en corderos de pelo finalizados en corral usando una dieta integral a base de grano sin fibra de forraje.

\section{Material y métodos}

Todos los procedimientos implicados en el manejo y sacrificio de los animales se realizaron de acuerdo a Normas Oficiales Mexicanas de SAGARPA (NOM-051-ZOO-1995: Cuidado humanitario de los animales durante la movilización, y NOM-033-ZOO-1995: Sacrificio de animales domésticos y salvajes). El estudio consistió de una prueba de comportamiento en corral que duró 30 días en el rancho "El Tilzapote", ubicado en el poblado de Ayutita, municipio de Autlán de Navarro, Jalisco. Posteriormente, se sacrificó la mitad de corderos en un rastro comercial ubicado en la cabecera municipal Tapalpa, Jalisco.

\section{Animales, alojamiento y manejo pre-experimental}

Se utilizaron 24 corderos enteros de la raza Katahdin, los cuales tenían una edad de 4 meses y un peso promedio al inicio de la prueba en corral de $35.8 \pm 5.3 \mathrm{~kg}$. Los corderos se alojaron en corraletas individuales y se adaptaron a una dieta base formulada para una ganancia diaria de peso de $300 \mathrm{~g}$ (Cuadro 1) ${ }^{(13)} 15$ días antes de la prueba. Adicionalmente, se desparasitaron cutáneamente con $1 \mathrm{ml}$ de ivermectina al 1\%/25 kg de peso vivo. La dieta se elaboró en una sola exhibición y dos muestras de alimento fueron tomadas de los diferentes costales para la determinación de los siguientes compuestos químicos: materia seca, proteína cruda, extracto etéreo, fibra total, fibra detergente ácida y neutra, y cenizas $^{(14,15)}$. También se calcularon con fórmulas ${ }^{(16,17)}$ los nutrientes digestibles totales y los diferentes tipos de energías (digestible, metabolizable, neta de mantenimiento y neta de ganancia). 
Cuadro 1: Ingredientes y composición química de la dieta base experimental

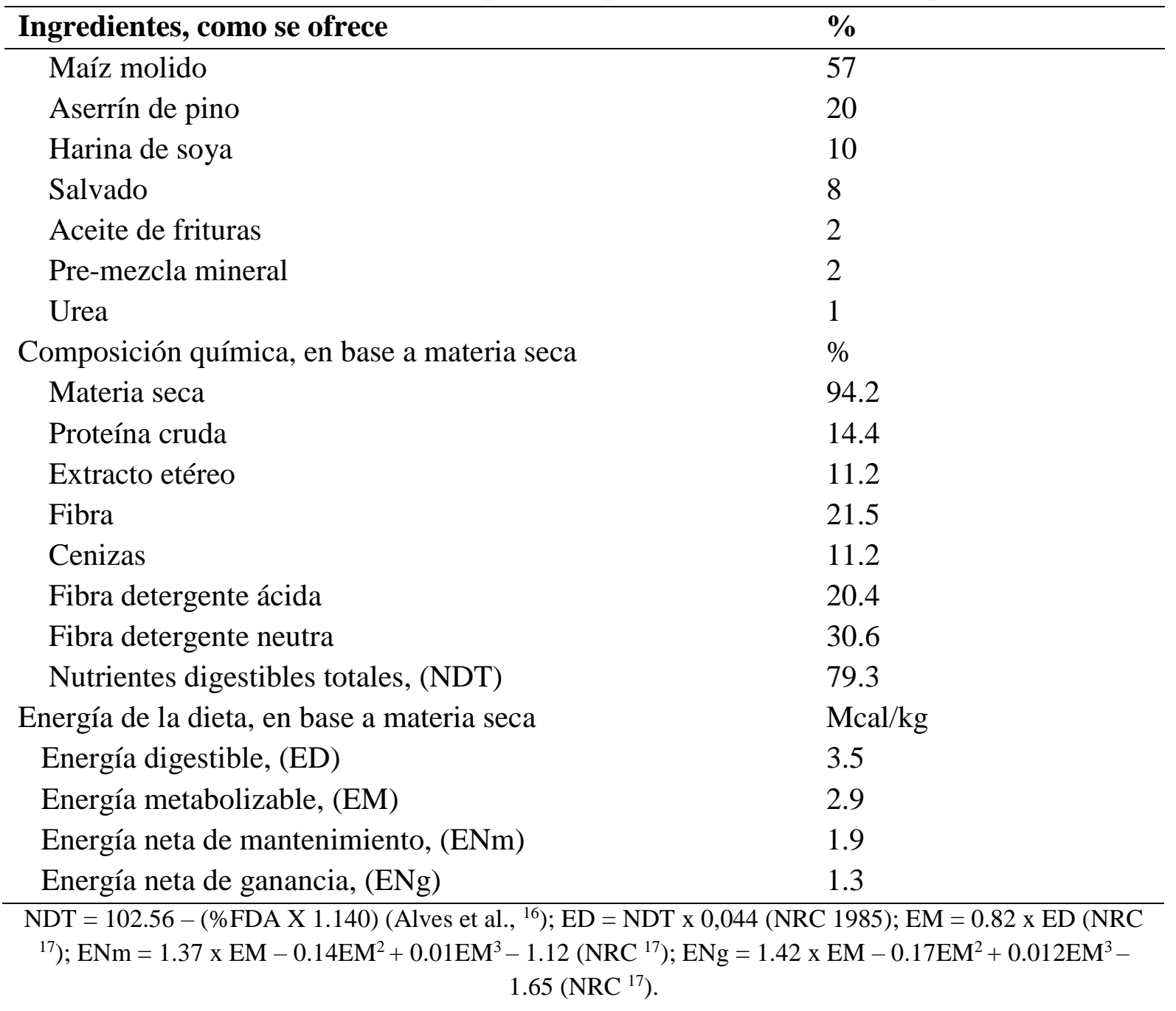

\section{Tratamientos y alimentación experimental}

El primer día de prueba, los corderos se pesaron y se formaron parejas de similar peso (factor de bloqueo) para asignarlos aleatoriamente dentro de cada bloque a uno de los dos tratamientos dietarios ( $\mathrm{n}=12$ ): 1) dieta basal sin $\mathrm{CZ}$ (testigo) y 2) dieta basal con $10 \mathrm{mg}$ de CZ genérico/d/cabeza (Grofactor®, Virbac México, Guadalajara, México). La dosis diaria de CZ se calculó basada en la cantidad de producto comercial $(208 \mathrm{mg}$ ) y se colocó en una cápsula de gel para ofrecerla oralmente a cada animal tratado con $\mathrm{CZ}$ antes de la alimentación de la mañana. En el caso del grupo testigo, a manera de placebo, una cápsula de gel que contenía $208 \mathrm{mg}$ de harina de soya fue ofrecida diariamente a cada cordero. La dosis ofrecida de CZ estuvo acorde a recomendaciones de estudios previos ${ }^{(18,19)}$. EL CZ se ofreció por 28 días, seguido de un periodo de retiro de $48 \mathrm{~h}$ antes de sacrificar los animales. 
La cantidad de alimento diario por corral se estimó para un rechazo aproximado del $10 \%$ y éste se ofreció en una sola exhibición a las 0700 h, justamente después de dar las cápsulas de tratamiento. La disponibilidad de agua fue ad libitum y todos los días se revisó el estado de salud de los animales por observación directa.

\section{Comportamiento productivo}

El comportamiento productivo se evaluó registrando el peso vivo al inicio (día 1) y final (día 31), antes de la alimentación en la mañana. La cantidad de alimento rechazado por corral también se registró. Con la información colectada se calculó el consumo diario de materia seca, la ganancia total, la ganancia diaria de peso y la eficiencia alimenticia del periodo completo (día1 a 30).

\section{Características de canal y cortes primarios}

Para la evaluación de la canal, solamente la mitad de los corderos de cada tratamiento $(\mathrm{n}=$ 6) se trasladó al rastro ubicado a $2 \mathrm{~h}$ del sitio de la prueba de comportamiento en corral. Dado que el estudio se realizó en un rancho privado, el productor definió que se sacrificaran los más pesados. En este sentido, se sacrificaron por el método de degüelle los corderos pertenecientes a los bloques del 7 al 12. El peso vivo individual de los corderos se registró nuevamente antes de entrar a línea de sacrificio. Los cuerpos de los corderos se evisceraron para registrar el peso de canal caliente (PCC), así como los pesos de cabeza, sangre, piel, corazón, pulmón, hígado, riñón, bazo, testículos y patas. Adicionalmente, se registró el peso de grasa mesentérica, omental y de riñón-pelvis-corazón (KPH por sus siglas en inglés). Posteriormente, las canales permanecieron por $24 \mathrm{~h}$ a $4^{\circ} \mathrm{C}$ y siguiendo las metodologías indicada por Avendaño-Reyes et $a l^{(18)}$ y Macías Cruz et al ${ }^{(20)}$, se registraron las siguientes variables: peso canal fría (PCF), área del músculo Longissimus dorsi (MLD) y espesor de grasa dorsal. También se registraron medidas morfométricas de la canal: largo de canal, pierna y paleta, asimismo circunferencia de tórax, cuello, pierna y paleta. Finalmente, la canal se cortó a través de la línea media y se registró el peso de la media canal derecha, la cual posteriormente se utilizó para obtener los siguientes cortes primarios: cuello, lomo, costilla con falda, pecho, pierna y paleta.

El peso vivo al sacrificio se ajustó al $96 \%$ por considerar que el contenido del tracto gastrointestinal era del $4 \%$. A excepción de grasa KPH, el peso de cada despojo de canal se expresó como un porcentaje del peso vivo al sacrificio. La grasa KPH se expresó como un 
porcentaje del PCC. El rendimiento en canal se calculó expresando el PCC como un porcentaje del peso vivo a sacrificio ajustado. La pérdida de canal por enfriamiento también se calculó expresando la diferencia entre PCC y PCF como un porcentaje del PCC. El rendimiento de cada corte primario se calculó expresando los respectivos pesos de cada corte como un porcentaje del peso de la media canal.

\section{Análisis estadístico}

El análisis estadístico consistió de un análisis de varianza bajo un diseño de bloques completamente al azar usando el procedimiento GLM del programa SAS ${ }^{(21)}$. Las medias se compararon con la prueba de Tukey a una $\alpha=0.05$. Tendencias no fueron consideradas dado el bajo número de repeticiones para variables relacionadas con sacrificio.

\section{Resultados}

En la prueba de comportamiento productivo solamente eficiencia alimenticia fue afectada $(P<0.01)$ por la suplementación de $\mathrm{CZ}$, siendo $9.4 \%$ mayor debido al AA- $\beta 2$ (Cuadro 2). El consumo de alimento tendió $(P=0.08)$ a disminuir por suplementación de $\mathrm{CZ}$. En características de la canal (Cuadro 3), la suplementación de $\mathrm{CZ}$ aumentó $(P \leq 0.02)$ en 7.5 , 7.3, 5.7 y $10.2 \%$ el PCC, el PCF, el rendimiento en canal y el área del MLD, respectivamente, pero también disminuyó $(P=0.05)$ el porcentaje de grasa KPH. El resto de las características de la canal fueron similares $(P \geq 0.17)$ entre tratamientos. En los pesos de despojos de la canal expresados como un porcentaje del peso vivo al sacrificio, la grasa mesentérica se redujo $(P<0.01) 23.2 \%$ por efecto del $\mathrm{CZ}$ dietario (Cuadro 4 ). Los pesos de los otros despojos no variaron $(P \geq 0.08)$ con la suplementación de CZ. Finalmente, la suplementación de $\mathrm{CZ}$ aumentó $(P \leq 0.04) 5.1 \%$ el rendimiento de lomo y pierna, pero redujo $(P=0.03) 8.1 \%$ el rendimiento de costilla con falda (Cuadro 5). Los demás cortes primarios tuvieron similares $(P \geq 0.18)$ rendimientos entre tratamientos. 
Cuadro 2: Comportamiento productivo de corderos de pelo suplementados con clorhidrato de zilpaterol genérico durante la etapa de finalización en corral

\begin{tabular}{lllll}
\hline & \multicolumn{2}{l}{$\begin{array}{l}\text { Clorhidrato de zilpaterol } \\
\text { (mg/cabeza/día) }\end{array}$} & \multicolumn{2}{l}{ Valor de } \\
\cline { 2 - 3 } Variables & $\mathbf{0}$ & $\mathbf{1 0}$ & & $\boldsymbol{P}$ \\
\hline Peso vivo, $\mathrm{kg}$ & & & & \\
$\quad$ Inicial & 35.7 & 35.8 & 0.2 & 0.83 \\
$\quad$ Intermedio & 41.5 & 41.2 & 0.3 & 0.49 \\
Final & 46.0 & 46.4 & 0.2 & 0.23 \\
Ganancia de peso total, $\mathrm{kg}$ & 10.3 & 10.6 & 0.2 & 0.29 \\
Ganancia diaria de peso, g/d & 343.0 & 354.3 & 7.1 & 0.29 \\
Consumo de alimento, $\mathrm{kg}$ & 1.7 & 1.5 & 0.1 & 0.08 \\
Eficiencia alimenticia, g/kg & 204.6 & 223.9 & 4.4 & $<0.01$ \\
\hline
\end{tabular}

Cuadro 3: Características y medidas morfométricas de la canal en corderos de pelo suplementados con clorhidrato de zilpaterol genérico durante la etapa de finalización en corral

\begin{tabular}{|c|c|c|c|c|}
\hline \multirow[b]{2}{*}{ Variables } & \multicolumn{2}{|c|}{$\begin{array}{l}\text { Clorhidrato de zilpaterol } \\
\text { (mg/cabeza/día) }\end{array}$} & \multirow{2}{*}{$\mathbf{E E}$} & \multirow{2}{*}{$\begin{array}{l}\text { Valor de } \\
P\end{array}$} \\
\hline & $\mathbf{0}$ & 10 & & \\
\hline Peso de canal caliente, $\mathrm{kg}$ & 22.7 & 24.4 & 0.2 & $<0.01$ \\
\hline Peso de canal fría, $\mathrm{kg}$ & 21.9 & 23.5 & 0.2 & $<0.01$ \\
\hline Rendimiento en canal, $\%$ & 49.1 & 51.9 & 0.4 & $<0.01$ \\
\hline Pérdida por enfriamiento, $\%$ & 3.3 & 3.5 & 0.2 & 0.52 \\
\hline Área del MLD, $\mathrm{cm}^{2}$ & 17.6 & 19.4 & 0.2 & 0.02 \\
\hline Espesor de grasa dorsal, $\mathrm{cm}$ & 0.3 & 0.2 & 0.1 & 0.33 \\
\hline Grasa KPH, \% & 4.6 & 3.3 & 0.4 & 0.05 \\
\hline Largo de canal, $\mathrm{cm}$ & 63.2 & 63.3 & 0.6 & 0.92 \\
\hline Circunferencia de tórax, $\mathrm{cm}$ & 74.5 & 75.2 & 0.5 & 0.36 \\
\hline Circunferencia de cuello, $\mathrm{cm}$ & 40.1 & 39.8 & 1.0 & 0.87 \\
\hline Largo de pierna, cm & 52.0 & 52.3 & 0.8 & 0.77 \\
\hline Circunferencia de pierna, $\mathrm{cm}$ & 43.5 & 45.7 & 1.0 & 0.19 \\
\hline Largo de paleta, cm & 41.7 & 41.0 & 0.8 & 0.59 \\
\hline Circunferencia de paleta, $\mathrm{cm}$ & 33.1 & 34.6 & 0.7 & 0.17 \\
\hline
\end{tabular}

$\mathrm{EE}=$ error estándar; $\mathrm{MLD}=$ Músculo Longissimus dorsi; Grasa KPH= La suma de las grasas acumuladas en riñones, pelvis y corazón. 
Cuadro 4: Pesos de los despojos de la canal expresados como porcentaje del peso vivo al sacrificio ajustado en corderos de pelo suplementados con clorhidrato de zilpaterol genérico durante la etapa de finalización en corral $(\%)$

\begin{tabular}{lllll}
\hline & \multicolumn{2}{l}{$\begin{array}{l}\text { Clorhidrato de zilpaterol } \\
\text { (mg/cabeza/día) }\end{array}$} & EE & \multicolumn{1}{c}{ Valor de } \\
\cline { 2 - 5 } Variables & $\mathbf{0}$ & $\mathbf{1 0}$ & 0.04 & 0.38 \\
\hline Cabeza & 3.27 & 3.33 & 0.12 & 0.26 \\
Sangre & 4.14 & 3.93 & 0.41 & 0.89 \\
Piel & 10.0 & 9.92 & 0.02 & 0.57 \\
Corazón & 0.48 & 0.48 & 0.12 & 0.96 \\
Pulmón & 1.73 & 1.74 & 0.17 & 0.08 \\
Hígado & 2.23 & 1.69 & 0.02 & 0.48 \\
Riñón & 0.31 & 0.28 & 0.02 & 0.08 \\
Bazo & 0.24 & 0.18 & 0.03 & $<0.01$ \\
Mesentérica & 0.99 & 0.76 & 0.15 & 0.72 \\
Omental & 2.45 & 2.54 & 0.04 & 0.74 \\
Testículos & 0.89 & 0.86 & 0.06 & 0.46 \\
Patas & 2.22 & 2.29 & & \\
\hline
\end{tabular}

$\mathrm{EE}=$ error estándar.

Cuadro 5: Rendimiento de cortes primarios en corderos de pelo suplementados con clorhidrato de zilpaterol genérico durante la etapa de finalización en corral

\begin{tabular}{|c|c|c|c|c|}
\hline \multirow[b]{2}{*}{ Variables* } & \multicolumn{2}{|c|}{$\begin{array}{l}\text { Clorhidrato de zilpaterol } \\
\text { (mg/cabeza/día) }\end{array}$} & \multirow{2}{*}{$\mathbf{E E}$} & \multirow{2}{*}{$\begin{array}{l}\text { Valor de } \\
P\end{array}$} \\
\hline & $\mathbf{0}$ & 10 & & \\
\hline Cuello, \% & 9.20 & 8.70 & 0.40 & 0.42 \\
\hline Lomo, \% & 19.20 & 20.17 & 0.26 & 0.04 \\
\hline Costilla con falda, $\%$ & 16.84 & 15.47 & 0.33 & 0.03 \\
\hline Pecho, \% & 5.56 & 5.07 & 0.22 & 0.18 \\
\hline Pierna, \% & 29.12 & 30.60 & 0.29 & 0.01 \\
\hline Paleta, $\%$ & 20.08 & 19.98 & 0.55 & 0.90 \\
\hline
\end{tabular}

*Todos los pesos de los cortes fueron expresados como porcentaje del peso de la media canal. $\mathrm{EE}=$ error estándar.

\section{Discusión}

La suplementación de $\mathrm{CZ}$ no resultó efectiva para mejorar el crecimiento, pero sí la eficiencia alimenticia de corderos finalizados con dietas sin fibra de forraje. Estos 
resultados difieren parcialmente con lo encontrado en estudios previos ${ }^{(5,22,23)}$, donde se reportó tanto mayor eficiencia alimenticia como ganancia de peso y peso final por suplementar este AA- $\beta 2$ durante un periodo de 30 días antes del sacrificio. No obstante, hay una serie de investigaciones indicando la ausencia del efecto de la suplementación de $\mathrm{CZ}$ en la ganancia diaria de peso y peso final de $\operatorname{corderos}^{(7,24,25)}$. Los corderos testigo fueron menos eficientes para aprovechar el alimento consumido porque la dieta tenía baja cantidad de fibra efectiva, dado que el aserrín estaba prácticamente molido. Esto pudo aumentar la tasa de pasaje gastrointestinal y disminuir la tasa de degradación de los microorganismos ruminales $^{(26)}$, aumentando el consumo de alimento y disminuyendo la eficiencia alimenticia. Así, la mejor eficiencia alimenticia observada en corderos alimentados con $\mathrm{CZ}$ se deba a que el AA- $\beta 2$ mejoró la digestibilidad de la dieta por aumentar la población ruminal de bacterias y reducir la motilidad del tracto gastrointestinal ${ }^{(27)}$. Así, los corderos tratados con CZ tendieron a disminuir su consumo diario de alimento sin afectar la tasa de crecimiento.

Si bien el crecimiento de los corderos no se mejoró con la suplementación de CZ genérico, la deposición de músculo aumentó significativamente por incluir este AA- $\beta 2$ como parte de la dieta. De tal manera que el PCC, el PCF, el rendimiento en canal y el área del MLD mejoraron con la suplementación del CZ, lo cual coincide con varios resultados de estudios $\operatorname{previos}^{(23,28,29)}$. Estos resultados se atribuyeron a que el $\mathrm{CZ}$ promovió una redirección de sustratos energéticos para incrementar la síntesis de proteína al mismo tiempo que redujo la proteólisis muscular ${ }^{(8)}$; ambas procesos conllevaron a una hipertrofia muscular en los corderos tratados con CZ. Inesperadamente, los resultados de este estudio mostraron que el origen del sustrato energético usado para la formación de músculo en los corderos suplementados con $\mathrm{CZ}$ fue principalmente a partir de tejido adiposo y no de órganos, viseras, cabeza, patas o testículos. Este hallazgo coincide con otras investigaciones en ovinos donde usaron $\mathrm{CZ}$ de la empresa Zilmax ${ }^{\circledR}{ }^{(6,18,28)}$, pero no con esas que suplementaron CZ de la empresa Grofactor ${ }^{\circledR(22,23)}$ como se hizo en este estudio. El CZ de Grofactor ${ }^{(22,23)}$ promueve en ovinos de engorda la formación de masa muscular por hacer uso de sustratos energéticos provenientes de una mejor repartición de energía dietaria, así como de algunos órganos, viseras o cabeza, pero no a partir de tejido graso. No se encontró una explicación respecto a porque cambió la forma de actuar del CZ en el presente estudio; aunque sí existen evidencias en bovinos de carne que este tipo de CZ remueve tejido graso para la formación de músculo ${ }^{(9)}$. No obstante, los resultados sugieren que el tipo de fibra usada en la dieta de finalización de corderos de engorda, puede modificar el modo de acción de este AA $\beta 2$. Se requiere hacer investigaciones a nivel de metabolismo y cinética ruminal para dilucidar cómo el tipo de fibra dietaria modifica el mecanismo de acción del CZ. 
Las medidas morfométricas de la canal no fueron afectadas por el $\mathrm{CZ}$ genérico, aun cuando se esperaba por lo menos piernas más largas y con mayor circunferencia considerando que algunos estudios han reportado que el mayor desarrollo muscular que provoca este AA $\beta 2$ se vuelve evidente físicamente en esta región corporal ${ }^{(4,20)}$. En ovinos de pelo finalizados con diferentes dosis de $\mathrm{CZ}$ genérico en dos experimentos ${ }^{(22)}$, se reportó un aumento cuadrático en el largo de canal, profundidad de tórax y perímetro de pierna como la dosis ofrecida de CZ aumentó de 0 a $0.20 \mathrm{mg} / \mathrm{kg}$ de peso vivo. En corderos ${ }^{(24,28)}$ y corderas ${ }^{(19)}$ también han encontrado un aumento en el perímetro de pierna sin efecto alguno en otras medidas morfométricas a consecuencia del $\mathrm{CZ}$ de patente.

De acuerdo a la literatura, los resultados de efecto de $\mathrm{CZ}$ de patente o genérico en los rendimientos de cortes primarios son inconsistentes. En la actual investigación, el CZ genérico mejoró el rendimiento de pierna y lomo, coincidiendo parcialmente con hallazgos de otros estudios donde encontraron solo un aumento en el rendimiento de pierna debido a la suplementación del CZ genérico en corderos de engorda ${ }^{(22,23)}$. Otros estudios no encontraron cambios en los rendimientos de cortes primarios por efecto de la $\mathrm{CZ}$ de patente en corderos finalizados en corral $^{(7,28)}$. Los resultados de este estudio se atribuyeron al hecho que tanto en pierna como en lomo predominan fibras musculares tipo II, en las cuales hay una gran cantidad de receptores adrenérgicos $\beta 2$, sitios donde se unen los AA $\beta 2$ ofrecidos exógenamente en la dieta para llevar acabo su acción biológica ${ }^{(8,18)}$.

\section{Conclusiones e implicaciones}

Se concluye que la suplementación de $\mathrm{CZ}$ en corderos de engorda mejora la eficiencia alimenticia, el peso y rendimiento en canal, y el área del MLD, sin afectar la ganancia de peso vivo, cuando reciben dietas sin fibra derivada de un forraje. Adicionalmente, la suplementación de CZ mejora el rendimiento de lomo y pierna. Finalmente, la suplementación de $\mathrm{CZ}$ es una estrategia nutricional recomendable para aumentar la ganancia de peso en canal y el rendimiento de cortes primarios con importancia económica en corderos finalizados con dietas formuladas sin fibra de forraje.

\section{Agradecimientos}

Al MVZ. Ramón Andrade Mancilla por las facilidades prestadas en su rancho "El Tilzapote”. Además, se reconoce el apoyo financiero otorgado al primer autor por parte del 
Centro Universitario de la Costa Sur, de la Universidad de Guadalajara, en el marco del Proyecto P3e 2018 No. 239754 de investigación pertinente en ciencias agropecuarias y afines para el desarrollo regional.

\section{Literatura citada:}

1. SIAP. Estadísticas de la producción de ovinos del Servicio de Información Agroalimentario y Pesquero, SAGARPA. 2018. Disponible en: http://infosiap.siap.gob.mx/repoAvance_siap_gb/pecResumen.jsp. Consultado 1 Mar, 2019.

2. Muñoz-Osorio GA, Aguilar-Caballero AJ, Sarmiento-Franco LA, Wurzinger M, Cámara-Sarmiento R. Technologies and strategies for improve hair lamb fattening systems in a tropical region: A review. Rev Ecosist Rec Agropecu 2016;3(8):267-277.

3. Lopez-Carlos MA, Ramirez RG, Aguilera-Soto JI, Plascencia A, Rodriguez H, Arechiga $\mathrm{CF}$, et al. Effect of two beta adrenergic agonists and feeding duration on feedlot performance and carcass characteristics of finishing lambs. Livest Sci 2011;138(13):251-258.

4. Aguilar López EY, González Ronquillo M, Salem AZM, Partida de la Peña JAP. Use of zilpaterol hydrochloride in sheep feeding. In: Salem AZM, editor. Nutritional strategies of animal feed additives. Nova Science Publishers; 2013:115-117.

5. Macías-Cruz U, Álvarez-Valenzuela FD, Soto-Navarro SA, Águila-Tepato E, AvendañoReyes L. Effect of zilpaterol hydrochloride on feedlot performance, nutrient intake, and digestibility in hair-breed sheep. J Anim Sci 2013;91(4):1844-1849.

6. Ortiz Rodea A, Amezcua Barbosa M, Partida de la Peña JA, González Ronquillo M. Effect of zilpaterol hydrochloride on animal performance and carcass characteristics in sheep: A meta-analysis. J Appl Anim Res 2016;44(1):104-112.

7. Macías-Cruz U, Avendaño-Reyes L, Vicente-Pérez R, Álvarez-Valenzuela FD, Correa-Calderón A, González-Ríos $\mathrm{H}$, et al. Growth and carcass characteristics of lambs finished with zilpaterol hydrochloride in grazing alfalfa / Crecimiento y características de la canal de corderos finalizados con clorhidrato de zilpaterol en pastoreo de alfalfa. Rev Mex Cien Pecu 2016;7(2):243-252.

8. Mersmann HJ. Overview of the effects of beta-adrenergic receptor agonists on animal growth including mechanisms of action. J Anim Sci 1998;76(1):160-172.

9. Avendaño-Reyes L, Meraz-Murillo FJ, Pérez-Linares C, Figueroa-Saavedra F, Correa A, Álvarez-Valenzuela FD, et al. Evaluation of the efficacy of Grofactor, a beta- 
adrenergic agonist based on zilpaterol hydrochloride, using feedlot finishing bulls. J Anim Sci 2016;94(7):2954-2961.

10. Iñiguez-Covarrubias G, Díaz-Teres R, Sanjuan-Dueñas R, Anzaldo-Hernández J, Rowell RM. Utilization of by-products from the tequila industry. Part 2: potential value of Agave tequilana Weber azul leaves. Bioresour Technol 2001;77(2):101-108.

11. Guerra-Medina CE, Cobos-Peralta MA, Montañez-Valdez OD, Pérez-Sato M. Uso de aserrín de pino (Pinnus patula) como fuente de fibra en dietas para borregos en cebo. Trop Subtrop Agroecosys 2010;12(3):667-673.

12. Guerra-Medina CE, Montañez-Valdez OD, Ley-de Coss A, Reyes-Gutiérrez JA, Gómez-Peña JE, Martínez-Tinajero JJ, et al. Fuentes alternas de fibra en dietas integrales para ovinos en engorda intensiva. Quehacer Cient Chiapas 2015;10(1):3-8.

13. NRC. Nutrient requirements of small ruminants: Sheep, goats, cervids, and new world camelids; 2007.

14. AOAC. Official Methods of Analysis . 15th ed. Association of Official Analytical Chemists. Arlington, VA, USA. 1990.

15. Van Soest PJ, Robertson JB, Lewis BA. Methods for dietary fiber, neutral detergent fiber, and nonstarch polysaccharides in relation to animal nutrition. J Dairy Sci 1991;74(10):223-232.

16. Alves AR, Beelen PMG, de Medeiros AN, Neto SG, Beelen RN. Consumo e digestibilidade do feno de sabiá por caprinos e ovinos suplementados com polietilenoglicol. Rev Caatinga 2011;24(2):152-157.

17. NRC. Nutrient requirements of sheep. 6th ed. Washington, DC, USA: National Academy Press; 1985.

18. Avendaño-Reyes L, Macías-Cruz U, Álvarez-Valenzuela FD, Águila-Tepato E, Torrentera-Olivera NG, Soto-Navarro SA. Effects of zilpaterol hydrochloride on growth performance, carcass characteristics, and wholesale cut yield of hair-breed ewe lambs consuming feedlot diets under moderate environmental conditions. J Anim Sci 2011;89(12):4188-4194.

19. Dávila-Ramírez JL, Macías-Cruz U, Torrentera-Olivera NG, González-Ríos H, PeñaRamos EA, Soto-Navarro SA, et al. Feedlot performance and carcass traits of hairbreed ewe lambs in response to zilpaterol hydrochloride and soybean oil supplementation. J Anim Sci 2015;93(6):3189-3196.

20. Macías-Cruz U, Álvarez-Valenzuela FD, Torrentera-Olivera NG, Velázquez-Morales JV, Correa-Calderón, Robinson PH, et al. Effect of zilpaterol hydrochloride on feedlot 
performance and carcass characteristics of ewe lambs during heat-stress conditions. Anim Prod Sci 2010;50(10):983.

21. SAS institute. SAS/STAT: User's Guide Statistics Released. 2004.

22. Avendaño-Reyes L, Torrentera-Olivera NG, Correa-Calderón A, López-Rincón G, Soto-Navarro SA, Rojo-Rubio R, et al. Daily optimal level of a generic beta-agonist based on zilpaterol hydrochloride for feedlot hair lambs. Small Ruminant Res 2018;165:48-53.

23. Rivera-Villegas A, Estrada-Angulo A, Castro-Pérez BI, Urías-Estrada JD, Ríos-Rincón FG, Rodríguez-Cordero D, et al. Comparative evaluation of supplemental zilpaterol hydrochloride sources on growth performance, dietary energetics and carcass characteristics of finishing lambs. Asian-Australas J Anim Sci 2018;00(00):1-8.

24. Dávila-Ramírez JL, Macías-Cruz U, Torrentera-Olivera NG, González-Ríos H, SotoNavarro SA, Rojo-Rubio R, et al. Effects of zilpaterol hydrochloride and soybean oil supplementation on feedlot performance and carcass characteristics of hair-breed ram lambs under heat stress conditions. J Anim Sci 2014;92(3):1184-1192.

25. Estrada-Angulo A, Barreras-Serrano A, Contreras G, Obregon JF, Robles-Estrada JC, Plascencia A, et al. Influence of level of zilpaterol chlorhydrate supplementation on growth performance and carcass characteristics of feedlot lambs. Small Ruminant Res 2008;80(1-3):107-110.

25. Salinas-Chavira J, Arzola C, García-Castillo RF, Briseño DA. Approaches to the level and quality of forage in feedlot diets for lambs. J Dairy Vet Anim Res 2017;5(3):9697.

27. McIntyre AS, Thompson DG. Review article: Adrenergic control of motor and secretory function in the gastrointestinal tract. Aliment Pharmacol Ther 1992:6:125142.

28. Rojo-Rubio R, Avendaño-Reyes L, Albarrán B, Vázquez JF, Soto-Navarro SA, Guerra $\mathrm{JE}$, et al. Zilpaterol hydrochloride improves growth performance and carcass traits without affecting wholesale cut yields of hair sheep finished in feedlot. J Appl Anim Res 2018;46(1):375-379.

29. López-Carlos MA, Ramírez RG, Aguilera-Soto JI, Aréchiga CF, Méndez-Llorente F, Rodríguez $\mathrm{H}$, et al. Effect of ractopamine hydrochloride and zilpaterol hydrochloride on growth, diet digestibility, intake and carcass characteristics of feedlot lambs. Livest Sci 2010;131(1):23-30. 